\title{
Effect of post materials on the biomechanical behaviour of endodontically treated tooth
}

\author{
Ahmed A. Madfa ${ }^{1, ~}$, Fadhel A. Al-Sanabani1 ${ }^{1}$, Nasr H. Al-Qudaimi \\ ${ }^{1}$ Department of Conservative Dentistry, Faculty of Dentistry, University of Thamar, Dhamar, Yemen \\ ${ }^{2}$ Department of Pediatric Dentistry, Preventive Dentistry and Orthodontics, Faculty of Dentistry, University of Thamar, Dhamar, Yemen
}

\section{Email address:}

ahmed_um_2011@yahoo.com (A. A. Madfa)

\section{To cite this article:}

Ahmed A. Madfa, Fadhel A. Al-Sanabani, Nasr H. Al-Qudaimi. Effect of Post Materials on the Biomechanical Behaviour of Endodontically Treated Tooth. International Journal of Medical Imaging. Vol. 2, No. 3, 2014, pp. 54-58.

doi: 10.11648/j.ijmi.20140203.12

\begin{abstract}
Objectives: To study the stress distributions in maxillary central incisor restored with Ni-Cr custom-made, gold custom-made, prefabricated titanium and prefabricated glass fibre dental posts subjected to oblique occlusal load at $100 \mathrm{~N}$. Materials and Methods: Four three dimensional finite element models of a maxillary central incisor restored with $\mathrm{Ni}-\mathrm{Cr}$ (Model I), gold custom-made (Model II), prefabricated titanium (Model III) and prefabricated glass fibre (Model IV) dental posts were constructed and oblique loading of $100 \mathrm{~N}$ was applied. Stress analysing at the along the centre of the post and post-surrounding structure interfaces were computed. Results: The result of this study indicates that the clinical three-dimensional image provided information about the behaviour of teeth under function in all directions. The results obtained from a finite element model on the restored system contain information about the stress distribution of each component of the restoration. The maximum von Mises stress distribution at post center was recorded with Model I (38.6 $\mathrm{MPa})$, followed by Model III (22.4 MPa) and Model II (20.8 MPa) respectively; whereas the minimum amount of stress was noticed in Model IV (10.9 MPa). At post dentin interface, the highest von Mises stress distribution was noticed with Model I (29.8 MPa), followed by Model III (18.9 MPa) and Model II (18.8 MPa) respectively; whereas the smallest amount of stress was noticed in Model IV (10.16 MPa). Conclusions: Glass fibre posts generated the least amount of stress concentration at middle and apical part of the posts compared to other dental posts.
\end{abstract}

Keywords: Finite Element Model, Custom-Made Dental Posts, Prefabricated Dental Posts, Stress Analysis

\section{Introduction}

Restoration of an endodontically treated tooth is considered as challenge for most dental practitioners. Dental post is often used after root canal treatment when restoring a damaged tooth with extensive loss of coronal tooth structure. ${ }^{[1]}$ Post insertion should be avoided if adequate retention can be achieved from the remaining coronal tooth structure. ${ }^{[2]}$ Unfortunately, these teeth that have been restored endodontically have been shown to exhibit a significantly shorter service life when compared with vital teeth. ${ }^{[3]}$

Dental posts are a way of building up and thereby retaining coronal restoration, however, posts do not reinforce the roots of the tooth. The development of the different post and core systems goes along with a paradigm shift in the restorative philosophy of treatment. Traditionally, custom-made posts and cores were the system of choice, but today, prefabricated metal and non-metal posts, combined with resin cores are considered a viable alternative. The prefabricated post and core system is one of the most popular systems because it requires less time in the dentist's chair. ${ }^{[4]}$

Some authors assert that posts may interfere with the mechanical resistance of treated teeth, leading to an increased risk of damage to the remaining tooth structure. ${ }^{[2,5]}$ To date, there is still no agreement about what is the ideal dental post for restoring endodontically treated teeth. ${ }^{[5]}$ Therefore, this was selected as the theme of the current study.

Three-dimensional Finite element method (3D FEM) has been used for stress analysis of teeth. What is more, the 3D FE method shows internal stress enabling predictions to be made about potential failure. ${ }^{[1]}$ Therefore, the study was aimed at comparing the stress distributions in maxillary central incisor restored with $\mathrm{Ni}-\mathrm{Cr}$ and gold custom-made, prefabricated titanium and glass fiber dental posts. 


\section{Materials and Method}

\subsection{Modelling of a Maxillary Central Incisor}

A three-dimensional (3D) model of an adult maxillary central incisor was developed using a Computed Tomography (CT) scan image set. Using Mimics (Materialise NV, Belgium) and Hounsfield's Unit, 3D models of a maxillary central incisor were constructed along with its surrounding cortical and cancellous bones. Restoration methods for endodontically treated teeth such as dental posts, composite cores and dental crown, were modeled based on the geometry of the root using a 'Solid Works' (Dassault Systèmes, USA). The dimension of each component was based on the data from literature. ${ }^{[7-9]} \mathrm{A}$ periodontal ligament (PDL) was modeled based on a tooth root with a thickness of $0.25 \mathrm{~mm}{ }^{[10]}$, and was subtracted from the volume of the cortical and cancellous bone (Figure 1). Since any cement and cementum is very thin. Thus, they are ignored and considered as the part of dentine. ${ }^{[11]}$

\subsection{Mesh Generation}

The tetrahedral elements (C3D4) were used with fine meshes to obtain accurate data. As constant stress tetrahedral elements exhibit slow convergence, during pilot study, a tetrahedral mesh of 148465 was used after a revealed that the error remained below $0.1 \%$ for two mesh sizes of 148465 and 236906 (Figure 1).

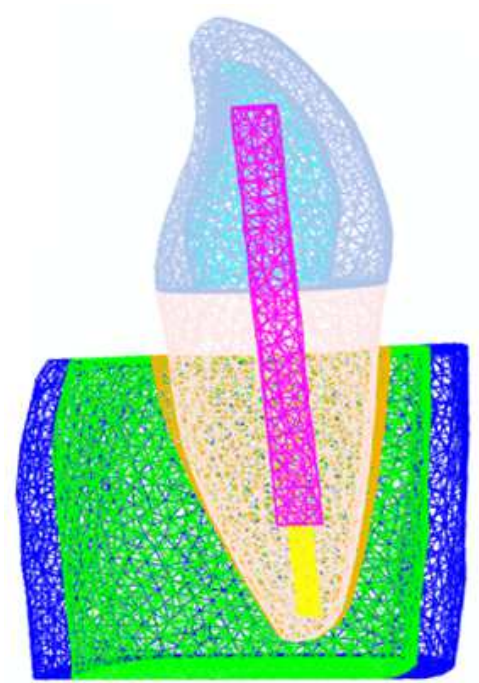

Figure 1. Schematic illustration of the three dimensional geometric model.

\subsection{Loads and Boundary Condition}

The boundary conditions for the nodes were placed along the bottom end line of the models and fixed to the supporting structure of the central incisor as prescribed by the system. An oblique load, angled at $45^{\circ}$, to simulate the masticatory force; $\mathrm{P}_{2}=100 \mathrm{~N}$ was chosen. ${ }^{[12]}$ All forces were applied on the aforementioned area as distributed pressure. Any stresses that are likely to be introduced during the endodontic treatment were neglected.

\subsection{Elastic Properties}

Mechanical properties of the restorative materials were created for each part of the FE model. The materials used for modeling each part of model tooth parts were assumed to be linearly. The elastic properties of $\mathrm{Ni}-\mathrm{Cr}$ custom-made (Model I), gold custom-made (Model II), prefabricated titanium (Model III) and glass fiber (Model IV) dental posts and other restorative materials used in the geometric model are presented in Tables 1 and 2.

Table 1. Mechanical properties of isotropic materials.

\begin{tabular}{lcc}
\hline \multicolumn{1}{c}{ Material } & Elastic modulus (MPa) & Poisson's ratio \\
\hline Cortical bone & 13700 & 0.3 \\
Cancellous bone & 1370 & 0.3 \\
Dentin & 18600 & 0.32 \\
PDL & 0.069 & 0.45 \\
Porcelain & 69000 & 0.28 \\
Gutta-percha & 140 & 0.45 \\
Composite resin & 12000 & 0.33 \\
Titanium post & 116000 & 0.33 \\
Ni-Cr alloy & 200000 & 0.33 \\
Gold alloy & 93 & 0.33 \\
\hline
\end{tabular}

Table 2. Mechanical properties of orthotropic materials.

\begin{tabular}{ll}
\hline Property & Glass fiber post \\
\hline Ex $(\mathrm{MPa})$ & 37000 \\
Ey $(\mathrm{MPa})$ & 9500 \\
Ez $(\mathrm{MPa})$ & 9500 \\
Vxy & 0.27 \\
Vxz & 0.34 \\
Vyz & 0.27 \\
Gxy & 3100 \\
Gxz & 3500 \\
Gyz & 3100 \\
\hline
\end{tabular}

\subsection{Finite Element Analysis}

The calculation of the von Mises stresses distributions and processing was carried out using ABAQUS/CAE software, Professional Version. Von Mises stresses were evaluated within the tooth, along the centre of the posts and at post and surrounding structures interfaces.

\section{Results}

The result of this study indicates that the clinical three-dimensional image provided information about the behaviour of teeth under function in all directions. The results obtained from a finite element model on the restored system contain information about the stress distribution of each component of the restoration.

In all the models the peak values of von Mises stresses stress was recorded at the middle third of the buccal aspect of the root surface. However, the smallest values were observed at level of both the apical portion of the post and the root apex.

The high stresses are also evidenced around the loading area and the outer surface of the dentine. Stress progressively decreases from the outer to the inner part of the root. The peak stress distribution was recorded with Model I followed by Model III and Model II respectively; 
whereas the minimum amount of stress was noticed in Model IV (Figures 2-5).
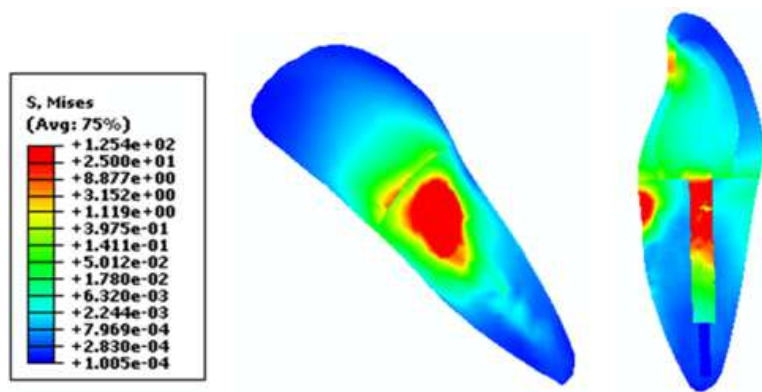

Figure 2. Contour plots for von Mises stress distribution in Model restored with Ni-Cr custom-made dental post.
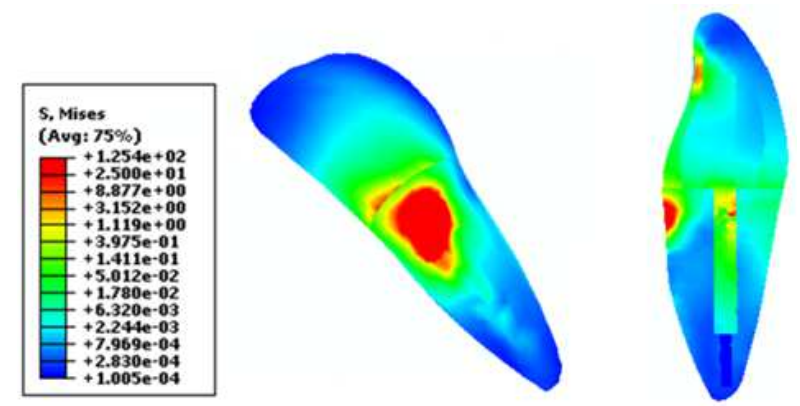

Figure 3. Contour plots for von Mises stress distribution in Model restored with gold custom-made dental post.
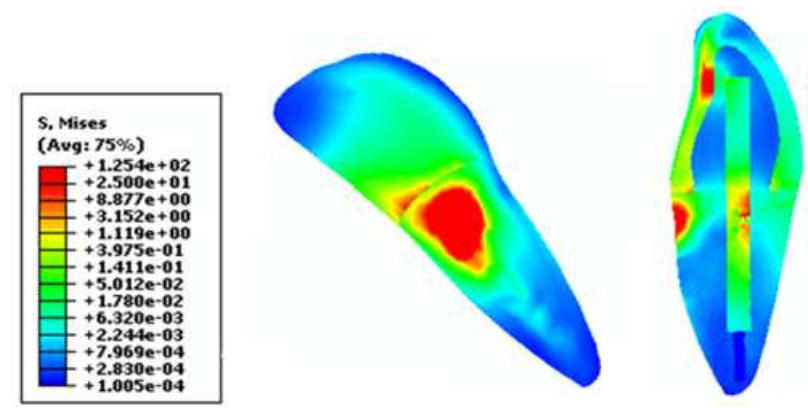

Figure 4. Contour plots for von Mises stress distribution in Model restored with prefabricated titanium dental post.
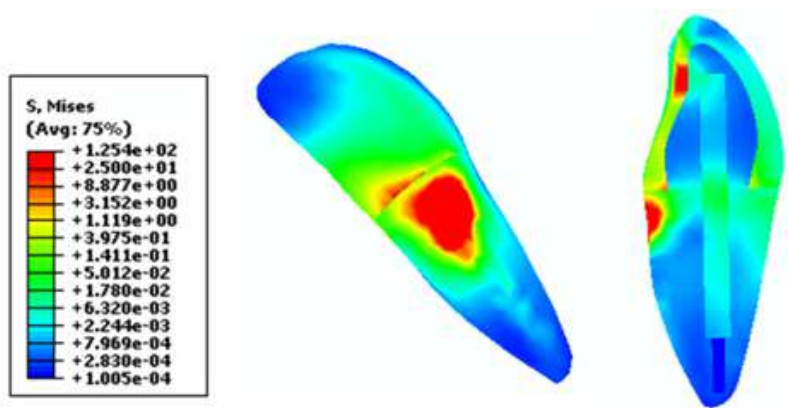

Figure 5. Contour plots for von Mises stress distribution in Model restored with prefabricated glass fibre dental post.

\subsection{Stress Distribution along the Center of the Posts}

Figure 6 exhibited the stress distribution inside the $\mathrm{Ni}-\mathrm{Cr}$ custom-made (Model I), gold custom-made (Model II), prefabricated titanium (Model III) and glass fiber (Model IV) dental posts. The maximum stress distribution inside the post is observed with the $\mathrm{Ni}-\mathrm{Cr}$ custom-made (38.6 MPa), followed by prefabricated titanium $(22.4 \mathrm{MPa})$ and gold custom-made (20.8 MPa) dental posts respectively; while the minimum amount of stress was observed in glass fiber posts $10.9 \mathrm{MPa}$ ) as shown in Figure 6.

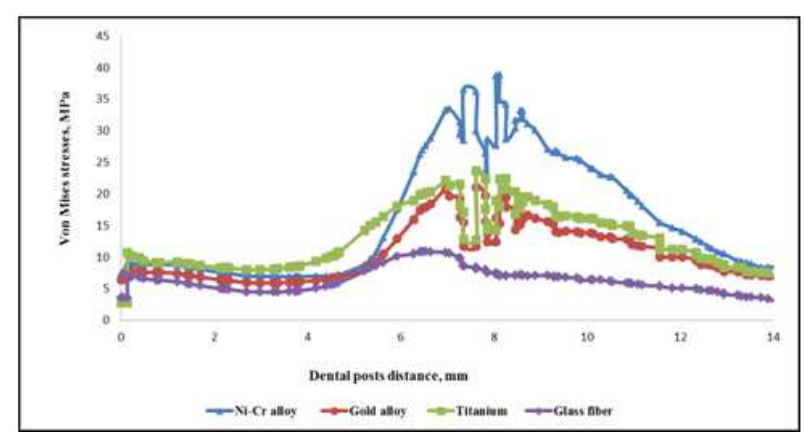

Figure 6. Von Mises stress distribution at the post centre.

\subsection{Stress Distribution at the Posts and Surrounding Structured Interfaces}

Figure 7 showed the stress distribution at post and surrounding structure interface. The highest stress distribution at the post and surrounding structure interface is observed with the $\mathrm{Ni}-\mathrm{Cr}$ custom-made (29.8 MPa), followed by prefabricated titanium (18.9 MPa) and gold custom-made dental posts respectively; while the smallest amount of stress $(18.8 \mathrm{MPa})$ was observed in glass fiber posts $(10.16 \mathrm{MPa})$.

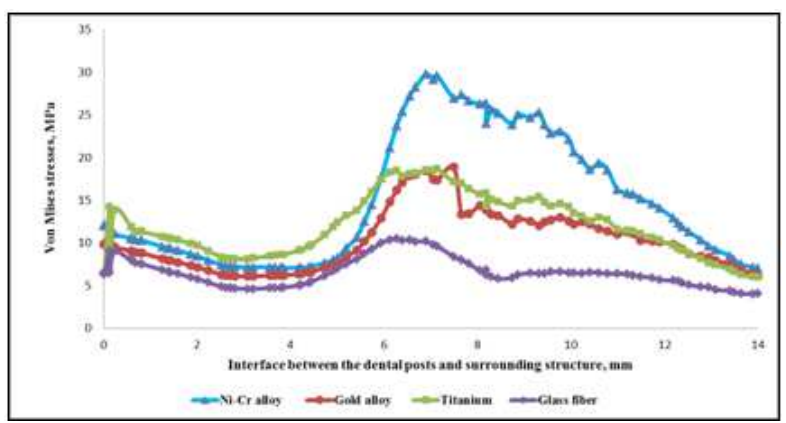

Figure 7. Von Mises stress distribution at the post and surrounding structures interface.

\section{Discussion}

Medical imaging can play a central role in the global healthcare system as it contributes to improved patient outcome and more cost-efficient healthcare in all major disease entities. Medical imaging techniques are a tool for mapping of anatomy and for detection and localization of a disease process. It produces important biological information about physiology, organ function, biochemistry, metabolism, molecular biology and functional genomics.

Finite element analysis is a powerful computational tool for modelling soft tissue deformation. It shows positive results for same-subject non-rigid image registration and for medical simulation applications. ${ }^{[13-15]}$

In the area of dentistry, studying dental structures and 
surrounding tissues in the oral cavity presents the basis for understanding the occurrence of pathological process and enables the correct approach and treatment. The success of restorative materials depends on their properties to withstand and resist occlusal forces and successfully support the remaining oral structure. ${ }^{[16]}$ FEA has been used to study internal stresses in teeth and different dental materials, and to optimize the shape of restorations. It eliminates the need for large number of experimental teeth due to variations in biological material properties and anatomy, mechanical testing involving biomaterials usually require a large number of samples. It has been used to represent simulated tooth mechanical behavior under occlusal loads in details. ${ }^{[17]}$

The reduction of stresses in the brittle material, like dentine, is advantageous as dentine can resist larger cyclic loading and chance of root failure is reduced. The reduction in the interface shear stress reduces the chances of the post loosing from dentine as the stress on bonding cement comes down.

FE method was used to study the stress generated in endodontically treated teeth which are that had been restored with a posts. It has been shown to be a useful tool when investigating complex systems that are difficult to be standardized during in vitro and in vivo studies. ${ }^{[18-20]}$

In this study, the model of the incisor was created from a CT scan image of an adult maxillary central incisor along with its surrounding structures. This provided a realistic rendition of clinical conditions. Toksavul et al. ${ }^{[11]}$ have omitted the cement layer. In this study, the cement layer was not included to simplicity the model.

It was observed that von Mises stresses were distributed along the posts, as expected, and that stress was concentrated on along the labial aspect of the posts, due to the direction of loading. However, higher stresses were observed $\mathrm{Ni}-\mathrm{Cr}$ custom-made, followed by prefabricated titanium and gold custom-made dental posts respectively; while the smallest amount of stress was observed in glass fiber posts. On the contrary, the stress distribution for glass fiber posts was concentrated mainly within the root dentine. Glass fiber post reduce the concentration of stress is likely to be due to a lower stiffness of this post. These results are in agreements with Joshi et al. [12] who reported that posts with high stiffness produced a created higher stress concentration compared to glass fiber posts. Posts with a higher elastic modulus have been found to cause amplification of stress within the post itself, yet a reduced stress distribution in the root dentin.

The results also agree with an earlier theoretical, in vitro investigation of the resistance of post-restored teeth to a cyclical loading ${ }^{[21]}$ and with a theoretical study using FE analysis. ${ }^{[22]}$ Silva et al. ${ }^{[23]}$ compared four different metal posts to glass fiber posts. They reported that the stress distribution within the tooth restored with a glass fiber post was more homogenous than those restored with metal posts. Furthermore, they found that stress tended to be concentrated at the outer boundary of metal posts. Asmussen et al. ${ }^{[24]}$ analyzed the stress distribution in endodontically treated teeth restored with glass fiber, titanium, and zirconia posts, reporting that an increase in the elastic modulus of the post caused a decrease in the stress within the dentin.

For the interface between the post and the surrounding structure, Ni-Cr custom-made post exhibited the highest stresses, while the smallest amount of stress was observed in glass fiber post compared to other types of post. Similarly, the results of this study revealed that glass fiber posts show a more balanced stress distribution at the post and dentine interface, as well as the cervical area of the tooth. This is agreement with Boschian et al. ${ }^{[25]}$, who reported that reinforced fiber glass composites distribute stress better than titanium or stainless steel posts. This concurs with the results of the present study which shows that metallic posts generate the highest stress values.

\section{Conclusions}

Glass fiber posts reduced the distribution of stress at the middle and apical parts of the post compared to other dental posts. However, Ni-Cr custom-made dental post increased the distribution of stress at the middle and apical parts of the post compared to other dental posts. Therefore, the chosen dental post with low elastic properties, practically when it is near to the elastic properties of dentine, is preferred in order to diminish the stresses in dentine and at the interface. This is in turn will resist larger cyclic loading and minimize the root failure and chance the post loosing from dentine.

\section{References}

[1] Morgano SM, Brackett SE. Foundation restorations in fixed prosthodontics: current knowledge and future needs. J Prosthet Dent (1999); 82: 643-57.

[2] Torbjörner A, Fransson B. Biomechanical aspects of prosthetic treatment of structurally compromised teeth. Int $\mathrm{J}$ Prosthodont (2004); 17: 135-41.

[3] Leempoel PJ, Kayser AF, Van Rossum GM, De Haan AF. The survival rate of bridges. A study of 1674 bridges in 40 Dutch general practices. J Oral Rehabil (1995); 22: 327-30.

[4] Baraban DJ. The restoration of endodontically treated teeth: an update. J Prosthet Dent 1988; 59:553-558.

[5] Ortega VL, Pegoraro LF, Conti PC, do Valle AL, Bonfante G. Evaluation of fracture resistance of endodontically treated maxillary premolars, restored with ceromer or heat-pressed ceramic inlays and fixed with dual-resin cements. J Oral Rehabil 2004; 31: 393-397.

[6] Ukon S, Moroi H, Okimoto K, Fujita M, Ishikawa M, Terada Y, et al. Influence of different elastic moduli of dowel and core on stress distribution in root. Dent Mater J 2000; 19: 50-64.

[7] Genovese K, Lamberti L, Pappalettere C. Finite element analysis of a new customized composite post system for endodontically treated teeth. J Biomech 2005; $38: 2375-2389$.

[8] Lanza A, Aversa R, Rengo S, Apicella D, Apicella A. 3D FEA of cemented steel, glass and carbon posts in a maxillary incisor. Dent Mater 2005; 21:709-715. 
[9] Santos AF, Tanaka CB, Lima RG, Espósito CO, Ballester RY, Braga RR, Meira JB. Vertical root fracture in upper premolars with endodontic posts: finite element analysis. $\mathrm{J}$ Endod (2009); 35: 117-20.

[10] Rees JS, Jacobsen PH. Elastic modulus of the periodontal ligament. Biomaterials 1997; 18: 995-999.

[11] Toksavul S, Zor M, Toman M, Güngör MA, Nergiz I, Artunç C. Analysis of dentinal stress distribution of maxillary central incisors subjected to various post-and-core applications; Oper Dent 2006; 31:89-96.

[12] Joshi S, Mukherjee A, Kheur M, Mehta, A. Mechanical performance of endodontically treated teeth. Finite Elem Anal Des 2001; 37:587-601.

[13] Azar F, Metaxas DN, Schnall MD. A deformable finite element model of the breast for predicting mechanical deformations under external perturbations. J Academic Radiol 2001; 8: 965-975.

[14] Brock K, Sharpe M, Dawson L, Kim S, Jaffray D. Accuracy of finite element model-based multi-organ deformable image registration. Med Phys 2005; 32: 1647-1659.

[15] Cash DM, Miga MI, Sinha TK, Galloway RL, Chapman WC. Compensating for intraoperative soft-tissue deformations using incomplete surface data and finite elements. IEEE Trans Med Imag 2005; 24: 1479-1491.

[16] Vasudeva G, Bogra P, Nikhil V, Singh V. Effect of occlusal restoration on stresses around class $\mathrm{V}$ restoration interface: A finite-element study. Indian J Dent Res 2011; 22: 295-302.

[17] Haiyan L, Jianying L, Zhenmin Z, Fok ASL. Fracture simulation of restored teeth using a continuum damage mechanics failure model. Dent Mater 2011; 27:e125-e133.
[18] Lanza A, Aversa R, Rengo S, Apicella D, Apicella A. 3D FEA of cemented steel, glass and carbon posts in a maxillary incisor. Dent Mater 2005; 21:709-715.

[19] Zarone F, Sorrentino R, Apicella D, Valentino B, Ferrari M, Aversa R, et al. Evaluation of the biomechanical behavior of maxillary central incisors restored by means of endocrowns compared to a natural tooth: a 3D static linear finite elements analysis. Dent Mater 2006; 22:1035-1044.

[20] Aggarwal S, Garg V. Finite element analysis of stress concentration in three popular brands of fiber posts systems used for maxillary central incisor teeth. J Cons Dent 2011; 14:293-6.

[21] Sahafi A, Peutzfeldt A, Ravnholt G, Asmussen E, Gotfredsen $\mathrm{K}$. Resistance to cyclic loading of teeth restored with posts. Clin Oral Investig 2005; 9:84-90.

[22] Ko CC, Chu CS, Chung KH, Lee MC. Effects of posts on dentin stress distributions in pulpless teeth. J Prosthet Dent 1992; 68:421-427.

[23] Silva NR, Castro CG, Santos-Filho PC, Silva GR, Campos $\mathrm{RE}$, Soares PV, et al. Influence of different post design and composition on stress distribution in maxillary central incisor: finite element analysis. Indian J Dent Res 2009; 20:153-158.

[24] Asmussen E, Peutzfeldt A, Sahafi A. Finite element analysis of stresses in endodontically treated, dowel-restored teeth. J Prosthet Dent 2005; 94:321-329.

[25] Boschian PL, Guidotti S, Pietrabissa R, Gagliani M. Stress distribution in a post-restored tooth using the three-dimensional finite element method. J Oral Rehabil 2006; 33:690-697. 